Presented at the 2002 Materials Research Society Spring Meeting, April 1-5, 2002, in San Francisco, California, and published in the Proceedings.

\title{
Materials for Solid State Lighting
}

\author{
S.G. Johnson \\ Lighting Research Group \\ Building Technologies Department \\ Environmental Energy Technologies Division \\ Ernest Orlando Lawrence Berkeley National Laboratory \\ University of California \\ 1 Cyclotron Road \\ Berkeley, California 94720 \\ J. A. Simmons \\ Sandia National Laboratory \\ P.O. Box 5800 \\ Albuquerque, New Mexico 87185
}

March 26, 2002

This work was supported by the Assistant Secretary for Energy Efficiency and Renewable Energy, Office of Building Technology, State and Community Programs, Office of Building Research and Standards of the U.S. Department of Energy under Contract No. DE-AC03-76SF00098. 


\title{
Materials for Solid State Lighting
}

\author{
S.G. Johnson ${ }^{1}$ and J. A. Simmons ${ }^{2}$
}

\section{Abstract}

Dramatic improvement in the efficiency of inorganic and organic light emitting diodes (LEDs and OLEDs) within the last decade has made these devices viable future energy efficient replacements for current light sources. However, both technologies must overcome major technical barriers, requiring significant advances in material science, before this goal can be achieved. Attention will be given to each technology associated with the following major areas of material research: 1) material synthesis, 2) process development, 3) device and defect physics, and 4) packaging.

The discussion on material synthesis will emphasize the need for further development of component materials, including substrates and electrodes, necessary for improving device performance. The process technology associated with the LEDs and OLEDs is very different, but in both cases it is one factor limiting device performance. Improvements in process control and methodology are expected to lead to additional benefits of higher yield, greater reliability and lower costs. Since reliability and performance are critical to these devices, an understanding of the basic physics of the devices and device failure mechanisms is necessary to effectively improve the product. The discussion will highlight some of the more basic material science problems remaining to be solved. In addition, consideration will be given to packaging technology and the need for the development of novel materials and geometries to increase the efficiencies and reliability of the devices. The discussion will emphasize the performance criteria necessary to meet lighting applications, in order to illustrate the gap between current status and market expectations for future product.

\section{Introduction}

Light sources used in general illumination have specific optical attributes, which include distribution, intensity, spectral properties and controllability. Other important product attributes include efficacy, lumen maintenance, product life and cost. Understanding these performance attributes and product requirements is necessary for an appreciation of the potential of solid state light sources to provide energy efficient products with improved product attributes. At this time, however, realizing this goal still requires significant advancements in materials related to this emerging technology.

Lighting systems are designed to distribute light to meet a myriad of different lighting applications we experience in our environment. The light sources used in these systems are typically characterized as either point sources or diffused sources. The solid-state lighting technology of light emitting diodes (LEDs) and organic light emitting diodes (OLEDs) conveniently fit these two descriptions, respectively. The LED is an intense point source that, with the proper optics, can be focused to provide high intensity beam distributions. Conversely, the OLED is much less intense per unit area and is more suited as a diffuse source. The ambient lighting of indoor spaces is generally provided by diffuse sources such as fluorescent luminaires. In good lighting practice the surface brightness of a diffuse source should be no greater than 850 candella per square meter to avoid glare; this is the surface brightness goal for which OLEDs should strive. Higher surface brightness will require diffusive elements. The light emitted from both LEDs and OLEDs must meet standards of quality and color; these are defined by the color-rendering index and color temperature of the light. Other performance attributes of particular importance are the efficacy of the product measured in lumens per watt, the maintenance of this light output as a function of time, the product life and, finally, the cost. The efficacy of standard incandescent lamps is, on average, 17

\footnotetext{
${ }^{1}$ S.G. Johnson is a Staff Scientist at Lawrence Berkeley National Laboratory, Berkeley, California.

${ }^{2}$ J.A. Simmons is a Staff Scientist Sandia National Laboratory, Albuquerque, New Mexico.
} 
lumens per watt, and that of fluorescent lamps varies from 60-100 lumens per watt. There is some depreciation in efficacy for these sources over their rated lifetimes of 1000 hours for incandescent lamps and 20,000 hours for fluorescent sources. These common light sources are a commodity product; hence, the market is very competitive and price sensitive. The current cost of solid-state sources is approximately 2 orders of magnitude higher than the sources being replaced, when measured in the cost per lumen. This currently limits their general application; however, as improvements in materials, processing, and efficiency are realized in the development of new products this cost differential will disappear.

\section{Technical Barriers to LEDs As a General Illumination Source}

LEDs, which we here take to mean inorganic LEDs, were first demonstrated in 1962 by Holonyak at General Electric [1]. They were commercially introduced in 1968 by Monsanto for use as indicators. At this stage, output levels were of order 1/1000 of a lumen, and the only color available was a deep red. During the 1970s steady progress in this new technology was made, resulting in LEDs of sufficient brightness to be observable in bright ambient light, and in a range of colors from red to a greenish-yellow. The materials used were primarily based upon GaAsP and GaP. In the 1980s the technology for producing heterojunctions in the AlGaAs/GaAs system enabled significant improvements in efficacy (lumens/W) for red LEDs, due to the ability to fabricate quantum wells for greater carrier confinement. It is only recently, in the 1990's, that two significant advances occurred which finally made it possible to envision the use of LEDs for general purpose illumination. First, researchers at Hewlett Packard [2] and Toshiba [3] developed LEDs from the quaternary alloy AlGaInP lattice matched to GaAs, enabling high brightness LEDs in the red to amber with efficacies in the range of 10's of lumens/W. The second and perhaps more important development was the demonstration by Nakamura [4] that LEDs could be grown from GaN-based materials, which for the first time enabled bright emission in the blue and green. This was a crucial development, since now white light sources could be realized based on the color mixing of different wavelengths, or the down conversion of blue to longer wavelengths using phosphors.

At present, three approaches have been envisioned for white light LEDs. These are: multi-chip LEDs, blue LEDs combined with one or more phosphors, and UV LEDs combined with multiple phosphors. (Of these, commercial products utilizing the last two approaches are currently available.) The use of multiple LEDs emitting at different colors will likely produce the most energy efficient white LED. With currently available LEDs, white light can be produced at an efficiency of about 30 lumens/W [5]. In order to achieve white, GaN and AlGaInP LEDs will need to be combined in a single package. However, since these different materials exhibit different temperature dependences and different aging rates, it is clear that such an approach will require a feedback circuit to maintain the right relative intensities of the constituent colors. This will increase the cost significantly. Nevertheless, the existence of independent controlling circuits for each color will enable a tremendous degree of control over brightness, color, and color temperature, and may be the preferred choice for the most demanding applications.

The second approach is to utilize a blue LED and one or more phosphors. The phosphors absorb a portion of the blue light, and then fluoresce at longer wavelengths such as yellow. The mixture of the remaining blue light and the fluoresced yellow light produces white. The drawback with this approach is a relatively poor coloring index, which can improve by adding additional phosphors in more colors. However, the color mixing issue is a serious problem: while the phosphors emit light isotropically, the blue light from the LED is emitted much more directionally, resulting in a variation of LED color with viewing angle. While this approach is the least costly, the color mixing issue is a serious one which must be overcome for the high quality light required for general illumination.

The third approach is a variation on the second. An ultraviolet (UV) LED is used to illuminate three or more different colored phosphors, with virtually all of the LED light absorbed. The phosphors are chosen to provide as high a color rendering index as possible. Because this scheme depends on all visible light being emitted by the phosphors, there is no issue of color mixing. Rather, the drawbacks to this approach 
reside with a lower efficiency due to the inherent energy loss in absorption of a UV photon and reemission of a lower energy visible photon. Further, the increased degradation of the packaging material and phosphor matrix material due to the high flux of UV radiation and high temperature is a serious issue.

The different approaches to white LEDs listed above are likely to all be used, depending on whether the application calls for highest quality light, maximum controllability, or greatest power efficiency. In any case, all three of the approaches will benefit from improvements in GaN-based materials, since it will be necessary to use these to produce the shortest wavelengths. The AlGaInN alloy system forms a continuous and direct bandgap semiconductor spanning ultraviolet to blue/green/yellow wavelengths. The efficiencies of current nitride-based LEDs are highest in the blue to near UV region, and decrease at longer wavelengths as the In component of the alloy increases.

Improvements in AlGaInN material are the single biggest material-related obstacle to white light inorganic LEDs. Despite the key role of this material, fundamental knowledge of its materials physics and growth chemistry is considerably less developed than that of more conventional III-V compound semiconductors such as GaAs. This is partly a result of the relatively recent advent of light emitting diodes in this material system, and partly due to its inherently unusual growth chemistry and the lack of native substrates.

A primary concern is the high number of defects in nitride materials. It is believed that the defects, including threading dislocations and point defects, provide centers for non-radiative recombination and contribute to the decline in efficiency of devices. Because no single-crystal GaN substrates of areas larger than $\sim 1 \mathrm{~cm}^{2}$ are available, growers have been using sapphire and $\mathrm{SiC}$ as substrates. This leads to epitaxial films with high defect densities, of order $10^{10} \mathrm{~cm}^{-2}$. Current areas of research include work to increase the size of native $\mathrm{GaN}$ or AlGaN substrates [6, 7], difficult due to the high temperatures and pressures required, as well as a number of approaches involving substrate patterning and selective area growth. Substrate patterning involves the nucleation of growth in selected regions, followed by lateral growth that allows the material to relax and grow with greatly reduced dislocation densities. Lateral epitaxial overgrowth (LEO), cantilever epitaxy, and pendeo-epitaxy have all extended the operating lifetime and output powers of $\mathrm{GaN}$ emitters [8], with cantilever epitaxy resulting in defect densities being lowered by a factor of 100 [9]. It is expected that these methods will play a major role in improving device efficiency and reliability. Of particular interest are Si substrates, due to the low cost and large areas available and the established industrial infrastructure. It may be that the selective area/ patterned substrate approach will eventually enable high quality nitride material to be grown on $\mathrm{Si}$, but more research is needed.

An additional area of research involves fundamental physics of point defects within the material, energy levels of ionized and neutral dopants, passivation of acceptors by $\mathrm{H}$, and diffusion models of the dopants. Dopant passivation from hydrogen is much more important for nitride material systems because of the large $\mathrm{N}-\mathrm{H}$ bond strength. One of the major barriers to high wallplug efficiencies is the difficulty in achieving good p-type doping of $\mathrm{AlGaN}$. The acceptor level is believed to move more deeply into the bandgap as the $\mathrm{Al}$ composition is increased. A fundamental understanding of the basic materials properties of the nitrides, particularly the behavior of acceptor levels, will help to set bounds on device designs and assist in optimizing performance by allowing one to take full advantage of known physical and electronic behavior.

Although power efficiency is clearly one of the major drivers for solid-state lighting materials research, reductions in cost are clearly equally important. Even if highly efficient proof-of-principle devices can be demonstrated, AlGaInN-based LEDs will not penetrate the market place if they cannot be manufactured in large quantities and at an acceptable cost. Chemical vapor deposition (CVD) growth technology of $\mathrm{GaN} / \mathrm{InGaN} / \mathrm{AlGaN}$ based materials is in a state of infancy compared to the sophisticated capabilities of metal-organic CVD (MOCVD) growth of other compound semiconductors. This difficulty is due in large 
part to the significantly different and poorly understood chemical interactions that exist for AlInGaN growth. Epitaxial growth of these materials is especially demanding, requiring deposition at low (500 C) and high $(1150 \mathrm{C})$ temperatures. The precursor gases are believed to react before reaching the substrate, causing severe difficulties in controlling growth rates and composition. This leads to undesirable effects such as highly reduced growth rates when Al-containing precursors are added to the inlet mix, unreliable doping which presumably is due to pre-reactions, and unpredictable behavior when one changes an inlet design, shape, or size. As a result, each AlInGaN -based CVD reactor becomes a one-of-a-kind apparatus that may take months to adjust through trial-and-error experiments before device-quality material can be grown. Enormous efforts have been made to build a scalable, reliable, AlInGaN CVD reactor using mostly Edisonian approaches. Such efforts have led to limited success. Clearly, there is a need to support these empirical efforts with science-based methods designed to understand the fundamental issues and to develop new reactor designs based on this understanding. Improved growth chemistry is vital for improving the material quality and for the eventual large-scale low-cost implementation of solid state lighting.

Recently, several research groups have reported improved material quality of AlInGaN -based thin film growth by depositing at atmospheric pressures and higher. This places additional strains on the development of a scalable AlInGaN reactor design. At these high pressures, buoyancy-driven convective instabilities become severe and difficult to control. These instabilities become more severe as the reactor is scaled to larger dimensions. Reliable manufacturing of AlInGaN materials will not be possible if buoyancy-driven instabilities are not understood and brought under control.

An additional research area of great importance to solid-state lighting is that of in situ monitoring and control of growth processes. Much of the cost in thin film manufacturing of complex optoelectronic devices goes into calibration of the deposition system. Typically, one grows test structures and uses ex situ methods to determine whether the growth rates and doping levels are correct. In situ monitors can shorten this calibration process considerably by directly measuring growth parameters. A number of new monitoring techniques are under investigation, including reflectivity-correcting pyrometers for measuring temperature, and laser-deflection measurements for sensing buoyancy instabilities.

As discussed, two of the approaches to solid state lighting involve the use of phosphors, or other photonconversion techniques. Commercial fluorescent lamps use phosphors to convert the UV radiation in the mercury discharge into visible light. Much effort over the years has gone into developing phosphors for fluorescents with high photon conversion efficiencies and good color rendering properties. Present technology uses a tricolor blend of phosphors, which unfortunately will be unsuitable for solid state lighting. The mercury discharge in fluorescents has its major line at $254 \mathrm{~nm}$, and existing phosphors have been engineered to absorb efficiently at this wavelength. They do not absorb nearly as well at the wavelengths needed for SSL-380 - $410 \mathrm{~nm}$ for UV LEDs, and 450-470 for blue LEDs. Clearly, the identification and optimization of a multi-color blend of phosphors, which absorb and reemit efficiently and yield good color rendering properties is of prime importance and a major task.

One alternative approach may be the use of nanoclusters. These are chemically synthesized nanometer sized particles of semiconductors, whose band-gap depends on their size due to quantum confinement effects in both the conduction band (electrons) the valence band (holes). The direct bandgap II-VI semiconductors, such as CdS, are of particular interest since high quantum yields of visible light may be possible. The peak of the light emission can be shifted from about $430 \mathrm{~nm}$ to nearly $700 \mathrm{~nm}$ by variation of the size of the nanoclusters, and/or surface treatment [10]. A solution of CdS nanoparticles with an external overcoat layer of $\mathrm{ZnS}$ has been shown to increase its fluorescence by a factor of 3 over that of uncoated CdS nanoparticles of the same size. Thus nanoclusters may provide a unique capability of engineering particular photon conversion parameters through both size and surface treatment. 
Up until now we have mainly discussed nitride materials issues; this is because red LEDs based on $\mathrm{AlGaInP}$ are a fairly mature technology for longer wavelengths. The $\left(\mathrm{Al}_{\mathrm{x}} \mathrm{Ga}_{1-\mathrm{x}}\right)_{0.50} \mathrm{In}_{0.50} \mathrm{P}$ material system is lattice matched to GaAs and is direct bandbap in nature over the range $\mathrm{x}=0$ to 0.53 , corresponding to $650 \mathrm{~nm}$ (deep red) to $555 \mathrm{~nm}$ (yellow-green). Internal quantum efficiencies approaching $100 \%$ can be achieved in this material in the red. However, because the GaAs substrate has a smaller energy gap, it absorbs much of the light produced by the AlGaInP active region. One approach that circumvents this difficulty is the growth of distributed Bragg reflector (DBR) mirrors of layered semiconductors between the substrate and the active region. The DBR reflects the light back upward, away from the substrate, increasing the total amount of photons emitted. Another approach is to utilize flip-chip methods where by the substrate is removed. This can have the additional advantage of the device being bonded to a high thermal conductivity carrier, which serves to lower the operating temperature and further increase power efficiency.

Even if the absorption by the substrate is circumvented, an additional difficulty remains which is common to both phosphide-and nitride-based LEDs: light extraction efficiency [11]. This arises due to the high refractive indices of the material, which means that most of the light generated in the semiconductor is totally internally reflected at the semiconductor/air or semiconductor/epoxy interface, with only a narrow cone near to normal escaping. The reflected light rattles around in the device until it is absorbed.

Creative, low cost solutions to the light extraction efficiency problem are needed. One solution is chipshaping, whereby the semiconductor die is shaped into a truncated inverted pyramid (TIP), which causes light to be directed to the pyramid corners where it can escape. Using this technique, the highest efficiency LED device to date was obtained, yielding an external quantum efficiency (which includes light extraction efficiency) of 55\% at $650 \mathrm{~nm}$ [12]. Additional approaches may include surface texturing, or even the incorporation of photonic lattices within the LED so as to suppress lateral emission modes, leaving only the vertical ones normal to the interface [13].

Finally, we note that packaging will be of prime importance if SSL is to achieve not only the high efficiency goals that have been set, but also the low cost goals. Critical to this will be the development of larger die sizes that can be driven at larger current densities, maximizing the amount of light obtained per unit area of material. While conventional indicator LEDs used die sizes of $0.25 \mathrm{~mm}$ square and $5 \mathrm{~mm}$ packages with $220{ }^{\circ} \mathrm{C} / \mathrm{W}$ thermal resistance, newer high brightness LEDs are up to $1 \mathrm{~mm}^{2}$ in size, and use high power packages having a thermal resistance of only $15^{\circ} \mathrm{C} / \mathrm{W}$ [14]. In the latter, the die is solderattached to a submount to compensate for the coefficient of thermal expansion mismatch between the LED and the copper heat sink. In addition to matters of heat extraction and die size, the materials used to encapsulate the LED must be improved. Current LED encapsulants show degradation (epoxy yellowing) to exposure by both UV and blue LED light from nitride materials. Developing UV-resistant plastics and materials for encapsulation is a high priority.

In summary, the development of cost-competitive inorganic LEDs for white lighting will rely on the solution of a number of materials-related R\&D challenges. This span the spectrum all the way from engineering challenges such as low thermal resistance packaging, through substrate patterning, selective area growth, chip-shaping and surface texturing for light extraction efficiency, all the way to fundamental science issues such as the energies of point defects within GaN an AlN. How quickly the market is penetrated by inorganic solid state lighting will depend to a large degree to the effectiveness of the solutions that are developed to these issues.

\section{Technical Barriers To OLEDs As a General Illumination}

Organic light emitting diodes are an emerging technology being driven both commercially and technically by the demands of the display market. The experience developed to meet the demands of this market is accelerating the opportunity for application of OLEDs as a general illumination light source. To meet the rigorous demands of cost and performance required of general illumination sources will require materials 
research into the components, their performance during operation of the device, process development, and encapsulation to enhance performance.

The principal components that comprise an OLED device are the organic materials, the electrodes and the substrate. The fundamental backbone of the device is combination of electroluminescent organic compounds. Since the initial investigation of "small molecule" [15] and polymeric [16, 17] compounds, there has been a steady increase in performance for the conversion of the supplied power to light. Recently, a system emitting in the green portion of the spectrum has achieved internal quantum efficiencies approaching 90\% [18]. This work clearly demonstrates the potential for OELD sources to become an energy efficient replacement to existing lighting technology.

The improvement in device efficiency has been achieved, in part, by addressing one of the most significant barriers, spin-symmetry conservation, which potentially limited organic devices to an efficiency of $25 \%$. In spin-independent exciton formation the singlet and triplet states are formed in a ratio of 1 to 3, respectively, in agreement with experimental measurements on the Alq3 [19]. The fluorescent singlet state undergoes a rapid radiative transition to the ground state, while the phosphorescent triplet state is more likely to undergo a nonradiative transition, due to the longer radiative lifetime of the state, hence, limiting the efficiency of the device to the percentage of singlet states populated, or approximately $25 \%$. Solutions to overcome this barrier have included the introduction of phosphorescent dyes [20], phosphors composed of heavy metal organic complexes [21]. Both approaches add dopants to the device to deactivate the triplet state of the host material into a state of the dopant that has a higher phosphorescent efficiency. The high efficiency of the organic metallic compounds results from the introduction of spin orbit coupling by the heavy metal atom, resulting in relatively high phosphorescence efficiencies. The very high efficiency of the green OLED, noted earlier, which also uses a heavy metal complex, is attributed to direct excitation of the dopant. The addition of dopants may have a secondary effect in optimization of performance by separating the function of charge transport from emission. Materials can therefore be optimized according to function.

The single/triplet ratio of 1 to 3 does not appear to be strictly adhered to in polymer OLEDs, with measurements of ratios approaching 1 to 1 [22]. This ratio was further enhanced by incorporating a heavy metal, Pt, directly into the polymer chain [23], resulting in an increase in the singlet population to $57 \%$ for this system. This phenomenon was demonstrated to occur in the polymer system, but was not observed in the monomer analog. This suggests that the efficiency of the polymer OLEDs could be increased significantly without the requirement for dopants or phosphors, which could potentially phase separate over life in these devices.

As research continues to improve the efficiency of the devices, work is also ongoing to develop efficient emitters in all colors. The ultimate goal is to develop a white light source with good color quality characterized by a high color-rendering index. This goal is distinctly different from the motivation of the display industry, and merits individual attention. The approaches to achieving this goal parallel those being pursued in LEDs, where multiple colors are combined from separate sources to generate white light or alternatively, a blue source is used to excite phosphors that emitted in a complementary portion of the spectrum. In contrast to the narrow band emission from LEDs, the broad molecular emission characteristic of OLEDs is a positive attribute for the blending of multiple colors to provide a white light source with a continuous spectrum in the visible range and with high color rendering characteristics. OLEDs are also different from LEDs in that white light could come from a single source by blending of different compounds. White light has been proposed for OLEDs by blending blue emitting polymer blends [24] and demonstrated by blending a blue and orange emitting polymer blend [25]. Blue continues to be the most difficult portion of the spectrum for which to find efficient systems and is critical to the development of white light sources. Recent work with phosphors in small molecule systems has demonstrated a system with an external quantum efficiency of 5.7\% [26]. 
Significant improvements in increasing internal quantum efficiencies for both small molecule and polymer systems have been demonstrated in the previous discussion; however, similar increases have not been realized in improving the extraction efficiency of these devices. Presently, only $25 \%$ of the light generated within an OLED is emitted by the device. Attempts to improve this performance have included modifying the substrate by texturing the surface [27], adding microspheres to the surfaces [28], and shaping the substrate to add a designed optical component $[29,30]$. Structuring of the internal layers has also been studied to provide an internal lensing element that could potentially be incorporated in the processing of the device [31]. Despite these efforts, which show promise, considerable improvement in the extraction efficiency must be realized before OLEDs can challenge current light source technology.

The life of OLEDs remains a critical issue. To compete as a light source in the general illumination market will require a product life of tens of thousands of hours. The life criteria of displayed devices of ten thousand hours sets a lower limit of what is expected in general illumination market. End of life will be determined when the original luminance intensity of the device decreases to $70 \%$ of the original value, versus $50 \%$ as often referenced for display applications. As the product ages the color is not expected to change. Hence, products made from the combination of different colored sources will require that the relative intensity of the respective sources remain constant over life of the product. Unlike existing products using LEDs, there are few commercial products using OLEDs that can be used to give a baseline of product life or performance as a function of color.

In an OLED the organic molecules are sandwiched between two electrodes, one a metal cathode and the other a transparent Indium Tin Oxide (ITO) anode. The metal cathode utilizes low work function metals to facilitate the efficient injection of the electrons into the organic electron transport layer. The low work function metals, i.e. $\mathrm{Li}$ or $\mathrm{Mg}$, are vacuum deposited onto the organic layer and are covered with a less reactive highly reflective conductor, such as aluminum or silver, which also provides a partial protective barrier. The low work function metals are very reactive and particularly sensitive to $\mathrm{O} 2$ and $\mathrm{H} 2 \mathrm{O}$ penetration during life of the product. Reaction with these molecules is associates with the growth nonemissive areas, "dark spots", at the cathode-organic interface $[32,33]$. The sensitivity of the electrodes to the diffusion of oxygen or water into the device makes the development of good environmental packaging critical. The elimination of the reactive metals, without a sacrifice in device performance, could have major impacts on process speed and product reliability. A secondary function of the metal electrode is to serve as an optical component to reflect light toward the transparent anode and substrate.

ITO is uniformly used as the anode in OLEDs devices as the best transparent hole injection material available. Currently, the ITO is coated onto a rigid glass substrate that provides support to the device during processing and provides a very good environmental barrier. A limitation of glass is the fragility and weight of the material for devices having large surface areas. The use of thin glass and plastics as a flexible substrate is being pursued. There is a desire to develop flexible substrates for both weight and cost benefits. Flexible substrates could ultimately lead to high-speed roll-to-roll processing. ITO may have limited application under these process conditions due to its limited elasticity, as it forms cracks when bent.

Assuming that large surfaces will be used for the OLED devices of the future, high speed processing will be required to make these devices in a cost effective manner. A lightweight, low-cost material would be a plastic substrate; however, a significant barrier to using this material is the permeability of the films to $\mathrm{H}_{2} \mathrm{O}$ and $\mathrm{O}_{2}$, which will degrade the cathodes. Work is ongoing to develop barrier layers that will be sufficiently impenetrable to provide a multi-year shelf life.

As the failure mechanism within the device becomes better understood, the size of the optimum emissive cell will be better defined. Cell size may be kept small to contain certain failure mechanisms, to improve the extraction efficiency, to improve the charge distribution across a large surface, or to control color. The formation of non-emissive areas at the cathode-organic interface has been a noted degradation process 
that tends to grow with time. This process could potentially be contained by segmenting the device into small units. In related thin-film devices shorts can occur between the electrodes; without self-healing electrodes, the small size of cells could prevent premature failure. If large surface area sources are developed it will be necessary to have uniformity of color and intensity across this surface. This may require some segmentation to ensure uniform charge density across the device. If the system combines colored cells to produce white light, multi-cell sources will be required. However, this may not significantly complicate the processing of the device. With the commercial development of OLEDs for display devices there is a great deal of work going on in refining process steps to provide a device with very well small and controllable colored cells. This level of sophistication will not be required for general illumination, but many of the lessons learned in this process will be applicable to OLED light sources. Given the very competitive nature of the display market, much of this knowledge resides as proprietary information within commercial concerns; however, general processing techniques have been defined. Vapor deposition is the common method for applying the organics in small molecule devices whereas spin coating is used in the laboratory to make experimental polymer devices. Manufacturing processing is moving toward methods that are reproducible, reliable, and have the potential of high speed. These techniques include inkjet printing and silk screening of the organic materials. Ultimately, it is expected that role-to-role processing techniques will be developed to make these devices.

Finally the entire system needs to be encapsulated in a process that is cost effective, that has a multi-year shelf life, and is stable with a low permeability to oxygen and water. And, the finished product, either rigid or flexible, should be neither fragile nor heavy.

\section{Conclusion}

The opportunity for LEDs and OLEDs to become the lighting technology of the future depends upon the solution of significant technical barriers involving the materials associated with these devices. Technical advances are required to continue to improve both the internal and external quantum efficiencies of these devices and to produce materials that will sustain performance over product life. Through a better understanding of the materials the challenges of processing, product life performance, and packaging can be met to make the these devices realize the promise of a more efficient light source with end user benefits not available today.

\section{Acknowledgment}

This work was supported by the Assistant Secretary for Energy Efficiency and Renewable Energy, Office of Building Technology, State and Community Programs, Office of Building Research and Standards of the U.S. Department of Energy under Contract No. DE-AC03-76SF00098.

\section{References}

1. Holonyak, N.J. and S.F. Bevaqua, Appl. Phys. Lett., 1962. 1: p. 82.

2. Kuo, C.P. et al., Appl. Phys. Lett., 1990. 57: p. 2937.

3. Sugawara, H., M. Ishikawa, and G. Hatakoshi, Appl. Phys. Lett., 1991. 58: p. 1010.

4. Nakamura, S. et al., Jpn. J. Appl. Phys., 1995. 34: p. L1332.

5. Jones, E.D., ed., Light Emitting Diodes (LEDs) for General Illumination, an OIDA Technology Roadmap. 2000.

6. Zauner, A.R.A., et al., J. Crystal Growth, 2000. 210: p. 435.

7. Schowalter, L.J. et al., Appl. Phys. Lett., 2000. 76: p. 985. 
8. Nakamura, S. in SPIE. 1999. San Jose, CA.

9. Ashby, C.I.H. et al., Appl. Phys. Lett., 2000. 77: p. 3233.

10. Wilcoxon, J.P. and G.A. Samara, Appl. Phys. Lett., 1996. 74: p. 3164.

11. Chui, H. et al., Semiconductors and Semimetals, 2000. 64: p. 69.

12. Krames, M.R. et al., Appl. Phys. Lett., 1999. 75: p. 2365.

13. Boroditsky, M. et al., Appl. Phys. Lett., 1999. 75: p. 1036.

14. Wierer, J.J. et al., Appl. Phys. Lett., 2001. 78: p. 3379.

15. Tang, C.W. and S.A. VanSlyke, Appl. Phys. Lett., 1987. 51: p. 913.

16. Burroughes, J.H., et al., Nature, 1990. 347: p. 539.

17. Braun, D. and A.J. Heeger, Appl. Phys. Lett., 1991. 58: p. 1982.

18. Adachi, C., et al., Nearly 100\% internal phosphorescence efficiency in an organic light-emitting device. Journal of Applied Physics, 2001. 90(10): p. 5048-5051.

19. Baldo, M.A., et al., Excitonic singlet-triplet ratio in a semiconducting organic thin film. Physical Review B-Condensed Matter, 1999. 60(20): p. 14422-14428.

20. Hoshino, S. and H. Suzuki, Appl. Phys. Lett., 1996. 69: p. 224.

21. Baldo, M.A., M.E. Thompson, and S.R. Forrest, High-efficiency fluorescent organic light-emitting devices using a phosphorescent sensitizer. Nature, 2000. 403(6771): p. 750-753.

22. Cao, Y., et al., Improved quantum efficiency fo electroluminescence in semiconducting polymers. Nature, 1999. 397: p. 414-417.

23. Wilson, J.S., et al., Spin-dependent exciton formation in pi-conjugated compounds. Nature, 2001. 413(6858): p. 828-831.

24. Thompson, J., et al., White light emission from blends of blue-emitting organic molecules: A general route to the white organic light-emitting diode? Applied Physics Letters, 2001. 79(5): p. 560-562.

25. Tasch, S., et al., Appl. Phys. Lett., 1997. 71: p. 2883.

26. Adachi, C., et al., Endothermic energy transfer: A mechanism for generating very efficient highenergy phosphorescent emission in organic materials. Applied Physics Letters, 2001. 79(13): p. 2082-2084.

27. Schnitzer, I. and E. Yablonovitch, Appl. Phys. Lett., 1993. 63: p. 2174.

28. Yamasaki, T. et al., Appl. Phys. Lett., 2000. 76: p. 1243.

29. Gu, G. et al., Opt. Lett., 1997. 22: p. 396.

30. Madigan, C., M.H. Lu, and J.C. Strum, Appl. Phys. Lett., 2000. 76: p. 1650.

31. Lupton, J.M., B.J. Matterson, and I.D.W. Samuels, Appl. Phys. Lett., 2000. 77(3340).

32. McElvain, J., et al., J. Applied Physics, 1996. 80: p. 6002.

33. Kolosov, D., et al., Direct observation of structural changes in organic light emitting devices during degradation. Journal of Applied Physics, 2001. 90(7): p. 3242-3247. 
